\title{
Diversité génétique et variabilité des caractères phénotypiques chez les arbres forestiers
}

\author{
A Kremer \\ Institut national de recherche agronomique, Laboratoire de génétique \\ et d'amélioration des arbres forestiers, \\ Pierroton, $B P$ 45, 39611 Gazinet Cedex, France
}

\begin{abstract}
Résumé - Une revue des résultats obtenus sur le niveau et l'organisation du polymorphisme génétique au sein des arbres forestiers est faite dans cette contribution. Elle s'attache à comparer les valeurs de la diversité moléculaire, principalement isoenzymatique (richesse allélique, diversité de Nei, coefficient de différentiation entre populations) à la variabilité génétique de caractères quantitatifs (héritabilité, coefficient de variation génétique, coefficient de différenciation). Les définitions et les relations entre les différents paramètres sont données en exergue. En moyenne, les espèces forestières manifestent des niveaux de diversité intrapopulation très élevés par rapport aux autres plantes. Les populations sont très différenciées pour les composantes et la phénologie de la croissance, les caractères liés à la structure de l'arbre (forme et architecture). À l'opposé, les différences de fréquences alléliques entre populations restent toujours très faibles, alors qu'au niveau multilocus une structuration géographique se dessine. Les niveaux de diversité et de variabilité atteints dans les populations sont interprétés à la lumière des tailles de population nécessaires à leur maintien : il est montré qu'une variation parallèle entre niveaux de diversité et de variabilité ne peut être observée que si les populations ont connu une réduction de leur taille à moins de quelques centaines d'individus. La concordance entre structuration de la diversité moléculaire et organisation de la variabilité génétique, observée dans les études faites sur l'ensemble de l'aire de répartition d'une espèce, résulte plutôt d'événements évolutifs anciens (bottlenecks, séparation des pools génétiques dans les populations refuges) que des effets récents et convergents de la sélection naturelle. Enfin ces résultats sont discutés dans le cadre d'une définition d'une politique de conservation des ressources génétiques forestières.
\end{abstract}

diversité génétique / variabilité génétique / différenciation génétique / arbre forestier / conservation des ressources génétiques

Summary - Genetic diversity and phenotypic variability of forest trees. This paper presents a review of results available on levels and distribution of polymorphism in forest trees. A comparison is made between parameters referring to genetic markers (allelic richness, gene diversity and genetic differentiation coefficient) and those addressing quantitative characters (heritability, coefficient of genetic variation and differentiation). 
Definitions and relationships between the different parameters are outlined. It is shown that forest trees exhibit higher levels of gene diversity than other plants. Populations are highly differentiated for traits related to growth and phenology, whereas allelic frequencies of gene markers show low variation among populations. Levels of diversity and variability are interpreted in relation to the population sizes necessary for their maintenance. Concordance between levels of diversity and variability is only expected when population sizes have been reduced to less than a few hundreds in their recent evolutionary history. In a wide range of studies of diversity and variability, similar trends of variation were found for quantitative traits and marker genes. These were attributed to evolutionary footprints (related to glacial refugia), rather than to recent and convergent selective pressures. These results are discussed in the framework of a policy for gene conservation of forest trees.

genetic diversity / genetic variability / genetic differentiation / forest tree / conservation of genetic resources

\section{INTRODUCTION}

Toute politique de conservation des ressources génétiques nécessite une description aussi exhaustive que possible de la diversité génétique dans les populations actuelles. Elle s'appuie aussi sur les mécanismes d'évolution de la diversité et sur leurs incidences sur le maintien de la diversité. La description repose sur 2 notions complémentaires : 1) le niveau de diversité à l'intérieur des populations, et 2) l'organisation de la diversité sur un ensemble de populations au sein d'une espèce donnée. L'objectif de cette contribution est de faire le point sur ces 2 notions chez les arbres forestiers, dont on rappellera en préambule leur évolution récente. Elle s'inspire, entre autres, des travaux présentés à 2 congrès récents (Müller-Starck et Ziehe, 1991 ; Adams et al, 1992), qui visaient à faire la synthèse sur les acquis obtenus par la génétique des populations des arbres forestiers.

Le premier écueil de cette entreprise se résume à la question suivante : quelle diversité utiliser? Historiquement, les généticiens forestiers se sont surtout intéressés à la description de la variabilité intraspécifique de caractères quantitatifs liés à l'adaptation, la croissance, la qualité de l'arbre (Wright, 1976). Les études de polymorphisme génétique basées sur les allozymes se sont développées plus récemment (Conkle, 1981). L'un des objectifs de cette contribution est de confronter ces 2 approches : d'une part en explicitant et en comparant les paramètres génétiques relatifs à chaque outil, d'autre part en confrontant les résultats obtenus par les 2 méthodes. Cette comparaison permet de dégager des éléments à prendre en compte pour la définition d'une politique de conservation des ressources génétiques. 


\section{ÉVOLUTION DES ESPÈCES FORESTIÈRES : RAPPEL HISTORIQUE ET SITUATION ACTUELLE}

\section{Histoire des populations forestières en zone tempérée}

Malgré leur origine très ancienne, la répartition actuelle des formations forestières dans l'hémisphère nord résulte d'une histoire récente. L'origine des coniferes remonte à 200 millions d'années alors que celle des feuillus date de 100 millions d'années (Thomas et Spicer, 1987). Ces grands groupes ont connu une différentiation spécifique importante au cours de l'Eocène ( -54 à -37 millions d'années) quand les zones tempérées actuelles connurent des climats tropicaux. Durant l'Oligocène, sous l'influence d'un refroidissement, les formations boréales et tempérées connurent à leur tour une spéciation importante en colonisant les zones tempérées actuelles; c'est la période au cours de laquelle la plupart des espèces feuillues actuelles se différencièrent.

La succession de périodes glaciaires et interglaciaires au cours du quaternaire se traduisit par des migrations de grande amplitude; les espèces furent repoussées vers les latitudes méridionales durant les périodes glaciaires et recolonisèrent l'Europe, l'Amérique du Nord et l'Asie au cours des périodes interglaciaires. La présence de barrières géographiques en Europe (Chaîne alpine et Méditerranée) a conduit à l'élimination de nombreuses espèces sur le continent européen principalement au cours des premières glaciations $(-2,4$ à $-1,7$ millions d'années). La majorité des genres était représentée par des espèces sur les 3 continents de l'hémisphère Nord à la fin du Tertiaire. À l'heure actuelle, la flore forestière ligneuse européenne est beaucoup plus pauvre en genres et en espèces qu'en Amérique du Nord. Les statistiques montrent qu'il existe au Nord des Alpes 5 espèces résineuses et 45 espèces feuillues. Ces chiffres sont respectivement de 23 et 148 en Amérique du Nord pour des latitudes analogues (Turner, 1993)

La glaciation la plus récente remonte à -18000 ans; depuis, les formations forestières ont progressivement recolonisé les parties septentrionales et nordiques de l'Europe. Les données palynologiques montrent que la migration s'est faite rapidement, $500 \mathrm{~m}$ par an dans le cas des chênes (Huntley et Birks, 1983). Ces données historiques suggèrent que la diversité génétique dans les populations actuelles a fortement été influencée par 2 facteurs :

- le nombre limité de populations refuges dans le Sud de l'Europe (Espagne, Italie, Grèce, Turquie) et certaines zones sous influence océanique, à partir desquelles la recolonisation s'est faite plus encore que le nombre, la taille de ces populations a dû largement moduler la diversité génétique; la restauration du polymorphisme, consécutive à une expansion démographique à partir d'effectifs initiaux limités, ne peut se faire qu'à l'issue de plusieurs centaines de générations (Lande et Barrowclough, 1987);

- la relative jeunesse des formations forestières du Nord de l'Europe; pour des espèces très longévives, qui par ailleurs se reproduisent végétativement par rejets de souche, le nombre de générations depuis la dernière glaciation n'est guère supérieur à 100 . Pour de nombreux paramètres génétiques (diversité et différenciation génétique), les valeurs asymptotiques résultant de l'équilibre entre les différents facteurs d'évolution (mutation, dérive, migration) n'ont pas encore été atteintes. 


\section{Effets limités de la domestication (Bary-Lenger et Nebout, 1993; Duby et. Wallon, 1975)}

Même si les forêts ont été exploitées par l'homme depuis le début de notre ère pour ses besoins domestiques (pâturage, panage, essartage) ou industriels (production de bois pour les forges, la marine, la sidérurgie, la construction), ces pratiques n'ont sans doute pas modifié de manière significative le niveau et l'évolution de la diversité génétique des arbres forestiers.

L'introduction de la sylviculture remonte au XII ${ }^{\mathbf{e}}$ siècle et a surtout été développée depuis le $\mathrm{XVI}^{\mathrm{e}}$ siècle. Elle a conduit à définir des règles culturales encore utilisées aujourd'hui, visant notamment à assurer le renouvellement des forêts par régénération naturelle après sélection phénotypique des semenciers. L'introduction de la sélection phénotypique dans la sylviculture n'a fait qu'accentuer la sélection naturelle elle-même. A titre d'exemple, les éclaircies effectuées dans les chênaies visaient essentiellement à éliminer les arbres les moins bien conformés, dont le houppier occupait l'étage dominé (éclaircies par le bas). De plus ces pratiques n'ont finalement concerné que 3 à 5 générations pour les espèces les plus longévives.

Les transferts de populations autochtones, déjà pratiqués au siècle dernier pour le chêne, et l'introduction d'espèces exotiques ont été très largement répandus au cours des 50 dernières années. Plus de 2 millions d'hectares de forêt ont ainsi été constitués en France entre 1950 et 1980 à partir de plantations d'espèces résineuses. Cette vague de reboisement s'est étendue aux espèces feuillues (chênes, hêtre, merisier, peuplier) plus récemment. Les conséquences génétiques de ces pratiques récentes («pollutions» génétiques des populations locales, adaptation des espèces introduites...) peuvent difficilement être appréciées à l'heure actuelle faute de recul suffisant.

Il résulte de cette analyse, qu'au plan génétique, les forêts européennes actuelles peuvent être classées en 2 grands ensembles :

- les formations d'origine autochtone, résultant des successions de régénérations naturelles aidées ou non par l'intervention humaine; il s'agit principalement des forêts feuillues de plaine constituées d'espèces à longue rotation (chênaie, hêtraie), des forêts feuillues ou résineuses d'altitude;

- les formations issues de transfert de populations et concernant des espèces autochtones ou exotiques (principalement nord-américaines) : ensemble des reboisements effectués en épicéas, pins, Douglas, etc, depuis un siècle.

À ces 2 ensembles, il faudra bientôt ajouter un troisième constitué des variétés sélectionnées dans le cadre de programmes d'amélioration génétique, concernant essentiellement les espèces économiquement importantes à courte ou moyenne rotation (épicéa, Douglas, pin maritime, pin sylvestre, peuplier, merisier).

\section{Populations d'arbres forestiers et pressions de sélection}

Dans les zones tempérées, les forêts ont été maintenues sur les terres impropres à l'agriculture où les conditions environnementales étaient nettement plus contraignantes (en altitude, sur les sols hydromorphes, calcaires). Elles sont, de ce fait, plus exposées aux accidents climatiques. Les dépérissements de forêts à la suite de ces accidents sont bien connus historiquement (Becker, 1984) et ont été abondamment 
commentés et illustrés à notre époque à la suite de l'augmentation de la pollution due à l'activité humaine. Les changements climatiques annoncés risquent par ailleurs de modifier en un temps record les formations forestières, ne permettant pas aux espèces de puiser dans leur réserve de diversité génétique pour s'adapter en moins d'une génération à ces nouvelles conditions.

\section{NIVEAU DE DIVERSITÉ GÉNÉTIQUE INTRAPOPULATION}

Les généticiens des populations et les généticiens quantitatifs utilisent des paramètres différents pour estimer la variabilité d'origine génétique au sein d'une population. Pour éviter toute confusion de vocabulaire, le polymorphisme des marqueurs moléculaires sera appelée diversité génétique par opposition à celui des caractères phénotypiques appelé variabilité génétique. La première notion fait essentiellement appel à l'étude des distributions des fréquences alléliques alors que la seconde s'appuie sur les composantes de la variance génétique de caractères quantitatifs.

\section{Paramètres de diversité et de variabilité génétique}

\section{Diversité génétique des marqueurs moléculaires}

Au niveau allélique, 3 paramètres de diversité sont généralement utilisés (Hartl et Clark, 1989). (Les paramètres définis au niveau génotypique ne seront pas évoqués ici) :

- le nombre d'allèles $(A)$ ou variants ailéliques identifiés dans la population, paramètre qualifié également de richesse allélique;

- la diversité génétique de Nei $(H)$, qui exprime la probabilité pour que 2 gènes tirés au hasard dans une population soient différents.

$$
H=1-\Sigma p_{i}^{2}
$$

où $p_{i}$ est la fréquence de l'allèle $i$ au locus considéré, $H$ est équivalent à l'hétérozygotie théorique sous l'hypothèse de Hardy-Weinberg;

- le nombre efficace d'allèles $\left(A_{e}\right)$ correspond au nombre d'allèles en tenant compte de leurs différences de fréquence $\left(A_{e}=1 /(1-H)\right)$.

Ces paramètres sont généralement estimés au niveau d'un seul locus à partir de marqueurs génétiques codominants. Dans le cas de plusieurs loci, la moyenne est prise sur tous les loci.

\section{Variabilité génétique de caractères phénotypiques}

Le niveau de variabilité au sein d'une population peut être estimé à partir des différentes composantes de la variance génotypique $\left(V_{A}, V_{D}\right.$ ou $V_{G}$ : respectivement variance additive, de dominance et génotypique). Ces composantes sont estimées dans des tests de descendances à partir des covariances entre apparentés. 
Dans le cas le plus simple d'un locus à 2 allèles, on peut montrer qu'il y a une liaison entre variabilité génétique et diversité (Kempthorne, 1957) :

$$
V_{A}=H a^{2}
$$

où $a$ est l'effet moyen de substitution des 2 allèles;

$$
V_{D}=H^{2} d^{2}
$$

où $d$ est l'effet de dominance généré par les 2 allèles;

$$
V_{G}=H\left(a^{2}+H d^{2}\right)
$$

où $H$ est la diversité génétique de Nei (équation 1 ).

Cette écriture n'est cependant pas généralisable au cas d'un locus à plusieurs allèles, bien que les composantes de la diversité interviennent dans l'expression des différentes variances. Par contre, elle peut être généralisée à un caractère dépendant de plusieurs loci, chacun d'entre eux restant biallélique. Elle permet cependant de préciser la nature de la variabilité génétique d'un caractère phénotypique qui dépend en fait de 2 facteurs :

- la diversité génétique proprement dite des loci intervenant dans le contrôle du caractère considéré et uniquement liée aux fréquences alléliques;

- l'effet proprement dit des allèles (effet additif ou de dominance).

Par opposition à la diversité génétique de Nei, ces variances ne sont pas bornées et dépendent de l'unité de mesure utilisée pour les caractères. Pour faciliter les comparaisons entre caractères ou entre espèces, 2 paramètres sont proposés :

- le coefficient de variation génétique défini au niveau des valeurs additives ou génotypiques;

- l'héritabilité au sens strict ou au sens large, qui correspond à la variance additive (ou génotypique) d'un caractère dont les valeurs ont été standardisées.

Comparaison des niveaux de diversité et de variabilité chez les arbres forestiers

\section{Niveau de diversité}

Une revue générale sur la diversité génétique (allozymique) des arbres forestiers basée sur des résultats obtenus sur 213 espèces appartenant à 54 genres montre que les espèces forestières sont extrêmement polymorphes (Hamrick et al, 1992; tableaux I et II).

Plus de $50 \%$ des loci sont polymorphes et le nombre moyen d'allèles par locus varie entre 1,83 et 1,68 alors que la diversité génétique est de l'ordre de 0,150. Il n'y a pas de différence de niveaux de diversité entre les gymnospermes et les angiospermes, malgré leur histoire ancienne très différente. Ces chiffres indiquent des niveaux de diversité nettement plus élevés par rapport à d'autres organismes : la diversité génétique $(H)$ est de 0,05 pour les vertébrés, de 0,11 pour les invertébrés et de 0,07 pour les plantes (Hartl et Clark, 1989). Les hypothèses les plus couramment invoquées pour interpréter ces résultats ont trait aux caractères biologiques des 
Tableau I. Niveau de diversité génétique intrapopulation chez les espèces forestières (Hamrick et al, 1992).

\begin{tabular}{lrccccc}
\hline Famille & $N$ & $n_{1}$ & $n_{2}$ & $A$ & $A_{e}$ & $H$ \\
\hline Gymnospermes & 102 & 8,9 & 17,3 & 1,83 & 1,20 & 0,151 \\
Angiospermes & 96 & 9,5 & 18,9 & 1,68 & 1,20 & 0,143 \\
\hline
\end{tabular}

$N$ : nombre d'espèces; $n_{1}:$ nombre moyen de populations par espèces; $n_{2}:$ nombre moyen de locus; $A$ : nombre moyen d'allèles par locus; $A_{e}$ : nombre efficace d'allèles $(=1 /(1-H)$; $H$ : diversité génétique de $\mathrm{Nei}(\mathrm{Nei}, 1987)$.

Tableau II. Paramètres de diversité génétique à l'intérieur de certains genres.

\begin{tabular}{lrcccc}
\hline Genre & $N$ & $A$ & $H$ & $G_{S T}$ & Référence \\
\hline Gymnospermes & & & & & \\
$\quad$ Abies & 7 & & 0,130 & 0,063 & $a$ \\
Picea & 28 & & 0,218 & 0,055 & $a$ \\
$\quad$ Pinus & 93 & & 0,136 & 0,065 & $a$ \\
$\quad$ Pseudotsuga & 11 & & 0,163 & 0,074 & $a$ \\
Angiospermes & & & & \\
$\quad$ Acacia & 13 & & 0,096 & 0,206 & $a$ \\
Eucalyptus & 16 & 1,89 & 0,174 & & $b$ \\
Populus & 10 & & 0,154 & 0,041 & $a$ \\
Quercus & 14 & 1,81 & 0,134 & 0,07 & $c$ \\
\hline
\end{tabular}

$N$ : nombre d'espèces étudiées; $A$ : nombre moyen d'allèles par locus; $H$ : diversité génétique de Nei (Nei, 1987); $G_{S T}$ : coefficient de différenciation génétique (Nei, 1987); $a$ : Hamrick et al, $1992 ; b$ : Moran, $1992 ; c$ : Kremer et Petit, 1993.

espèces forestières. En premier lieu, il faut citer les tailles de populations qui se chiffrent à plusieurs milliers, voire dizaines de milliers, pour les espèces sociales (Schoen et Brown, 1991), et le régime de reproduction qui est proche de l'allogamie stricte à l'exception d'un nombre limité d'espèces (Mitton, 1992). Ces propriétés biologiques permettent de prédire 30 à $40 \%$ des niveaux de diversité intrapopulation (Hamrick et al, 1992; Hamrick et Godt, 1990).

À l'intérieur d'une espèce donnée couvrant une large amplitude latitudinale (espèces résineuses de l'Ouest Américain), les populations méridionales manifestent généralement des niveaux de diversité plus élevés que les populations septentrionales (Ledig, 1988). Des résultats similaires ont été obtenus en Europe pour le hêtre (Comps et al, 1991) et le chêne sessile (Kremer et al, 1991). À l'opposé, les espèces ayant une distribution limitée aux zones boréales, sont plus polymorphes que les espèces des zones tempérées ou tropicales (Hamrick et al, 1992).

\section{Niveau de variabilité génétique}

La variabilité génétique de caractères quantitatifs n'a été étudiée de manière exhaustive que pour les espèces économiquement importantes et pour des caractères 
liés directement ou indirectement à leur valeur commerciale. Il s'agit essentiellement de la production en quantité de bois (volume du tronc), en qualité extrinsèque du bois (forme du tronc, architecture), en qualité intrinsèque du bois (densité du bois). Les estimations des valeurs des paramètres génétiques disponibles à ce jour (tableau III) se réfêrent à des populations d'amélioration de première génération. En d'autres termes, elles correspondent aux peuplements actuels, dans lesquels les populations d'amélioration ont été recrutées. Le recrutement s'est fait en général sur la base d'une sélection phénotypique en forêt, qui a pu entraîner une sous-estimation des paramètres de variabilité génétique.

Les caractères pour lesquels ces estimations sont disponibles sont tous des caractères composites résultant de l'interaction de très nombreuses composantes élémentaires. Ils dépendent sans doute d'un très grand nombre de loci, bien qu'aucune donnée ne soit disponible à ce sujet. Les valeurs d'héritabilité sont plus élevées pour les critères liés à la structure de l'arbre (forme, architecture, densité du bois) par comparaison à ceux liés à la vigueur (hauteur, diamètre). L'opposition est très nette entre la densité du bois et les autres caractères, quelle que soit l'espèce. Les caractères de vigueur et de forme interviennent directement dans la valeur adaptative de l'arbre. Les peuplements forestiers sont extrêmement denses (plusieurs centaines de milliers d'individus à l'hectare dans un régénération naturelle). Une supériorité en hauteur et en développement du houppier confère un avantage en terme de valeur adaptative. Ces résultats expérimentaux, bien que limités à un faible nombre de caractères, semblent corroborer les prédictions théoriques montrant que dans des populations à l'équilibre l'héritabilité des caractères liés à la valeur adaptative tend vers zéro (Mousseau et Roff, 1987; Price and Schulter, 1991). Les valeurs des coefficients de variation génétique (basés sur les valeurs additives) montrent une plus forte homogénéité entre espèces que les valeurs d'héritabilité pour les mêmes caractères.

Malheureusement, les données actuellement disponibles ne s'adressent en général qu'à une seule population par espèce, celle pour laquelle un programme de sélection a été engagé. Elle correspond à une forêt ou une région géographique limitée de l'aire de distribution d'une espèce.

\section{Comparaison entre niveau de diversité et niveau de variabilité intrapopulation}

L'écriture de la relation entre variabilité et diversité (équation 2) suggère l'existence d'une corrélation entre ces 2 paramètres. Malheureusement les données relatives à la diversité, disponibles à l'heure actuelle, ne concernent pas les locus contrôlant les caractères quantitatifs, mais des marqueurs génétiques neutres vis-à-vis de la sélection naturelle. Peut-on cependant, même en l'absence de toute liaison génétique entre loci impliqués dans les caractères phénotypiques d'une part et marqueurs d'autre part, rechercher une correspondance entre niveau de diversité et niveau de variabilité? Cette question conduit à comparer les effets des facteurs d'évolution sur le niveau de la diversité et de la variabilité dans les populations naturelles.

Pour un caractère quantitatif, nous nous plaçons dans le cadre de la théorie développée par $\mathbf{R}$ Lande $(1975,1976)$, qui considère que celui-ci est soumis à une sélection stabilisatrice favorisant les phénotypes intermédiaires. La part de la 


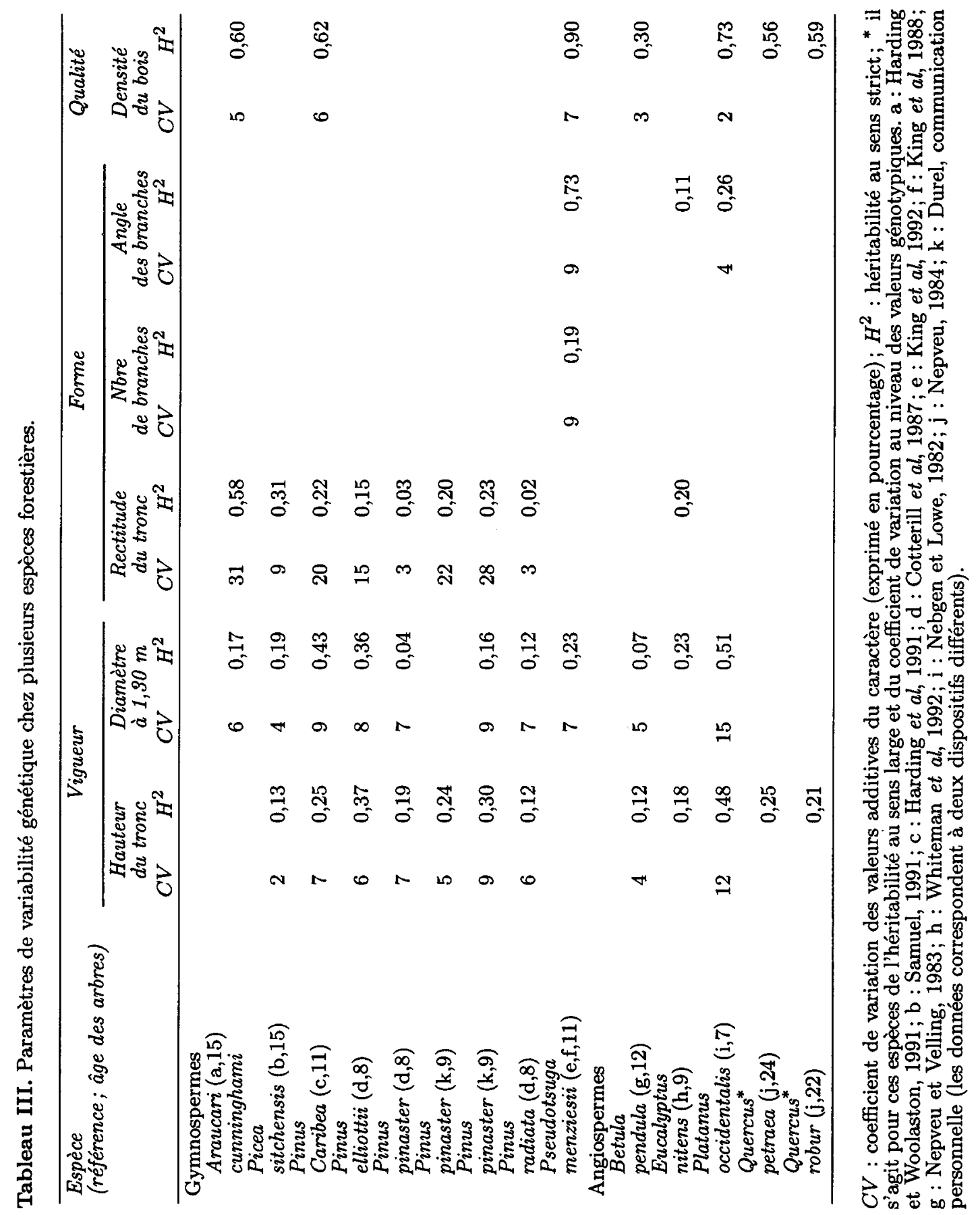


variabilité générée par la mutation est extrêmement élevée $\left(10^{-3}\right.$ à $10^{-2}$ de la variance additive, Lynch, 1988). Il en résulte que la perte de la variance due à la dérive reste négligeable devant celle due à la sélection stabilisatrice quand la taille de la population est supérieure à 500 individus (Lande et Barrowclough, 1987). À l'équilibre entre les 3 forces évolutives, pour un caractère dont l'héritabilité se situe entre 0,3 et 0,7 (tableau III), une population de 500 individus peut maintenir une variance presque aussi élevée qu'une population de taille infinie.

Pour un marqueur génétique neutre, le niveau de diversité à l'équilibre entre mutation et dérive est une fonction croissante de la taille de population (Hartl et Clark, 1989). Comme les taux de mutation sont nettement plus faibles $\left(10^{-5}\right.$ à $10^{-7}$ par génération pour les isozymes), les tailles de population nécessaires au maintien de la diversité doivent être nettement plus élevées que pour les caractères quantitatifs. Si l'on se base sur les données des niveaux de diversité relatives aux arbres forestiers (tableau II), en supposant en outre que ces valeurs correspondent à celle de l'équilibre entre mutation et dérive, les tailles de population requises pour maintenir ce niveau doivent être supérieures à plusieurs dizaines de milliers.

La comparaison de l'effet des tailles de population sur le niveau de diversité et de variabilité suggère, en quelque sorte une relation de type triangulaire entre les 2 paramètres : diminution parallèle de $H$ et de $V_{A}$ quand les tailles de populations sont faibles (inférieure à 500 individus), variation indépendante de $V_{A}$ et de $H$ quand les tailles sont élevées. Précisons enfin qu'il convient de prendre le chiffre de 500 comme un ordre de grandeur susceptible de varier selon les espèces.

Les arbres forestiers ont connu plusieurs vagues successives de colonisation dans l'hémisphère Nord, ayant largement modulé les tailles de population. Au cours de leur histoire récente, les modifications d'effectifs se sont accentuées dans certaines zones géographiques soumises à des accidents climatiques ou tectoniques (incendies de forêts, volcanisme). Les restrictions drastiques de tailles de populations à quelques centaines d'arbres affectent sans distinction les loci neutres ou soumis à sélection, comme nous venons de le voir dans le paragraphe précédent. Les populations ayant connu des bottlenecks dans leur histoire récente ne récupèrent une diversité importante qu'au terme de très nombreuses générations. Plusieurs exemples illustrent l'effet des réductions des tailles de populations sur le niveau de la diversité moléculaire et la variabilité des caractères phénotypiques. Pinus resinosa est une espèce couvrant le nord-est des États-Unis. Elle se singularise par une absence totale de diversité allozymique associée à une variabilité quasi nulle de la croissance et de la forme des arbres (Mosseler et al, 1991; Fowler and Lester, 1970). Thuya plicata, dont l'aire de distribution s'étend dans l'Ouest américain depuis la Californie jusqu'en Colombie britannique manifeste la même uniformité pour les 2 types de caractères (Copes, 1981). Ces 2 espèces occupent aujourd'hui de très vastes étendues, mais ont sans doute connu des bottlenecks au cours de leur passé récent. On peut noter à ce sujet que la récupération du polymorphisme consécutive à des bottlenecks et par expansion démographique de l'espèce est beaucoup plus rapide pour un caractère quantitatif que pour un marqueur moléculaire (Lande et Barrowclough, 1987). Elle se fait en quelques centaines de générations pour la variabilité génétique et quelques dizaines de milliers pour un marqueur moléculaire. 


\section{ORGANISATION DE LA DIVERSITÉ ET DE LA VARIABILITÉ GÉNÉTIQUES CHEZ LES ESPĖCES FORESTIÈRES : DIFFÉRENCIATION ENTRE POPULATIONS}

\section{Paramètres de différenciation entre populations}

\section{Paramètres de différenciation au niveau des marqueurs moléculaires}

La structuration génétique au sein d'un ensemble de populations a d'abord été décrite par les statistiques $F$ de Wright (1965) au niveau allélique. Elle a été généralisée au niveau d'un locus puis d'un ensemble de loci par Nei $(1973,1977)$. Le coefficient de différenciation génétique entre populations $\left(G_{S T}\right)$ est défini de la manière suivante au niveau d'un locus :

$$
G_{S T}=1-H_{S} / H_{T}
$$

où $H_{S}$ est la moyenne (sur toutes les populations) des diversités génétiques intrapopulations; $H_{T}$ est la diversité génétique sur l'ensemble des populations considérées comme une population unique (diversité totale).

Quand plusieurs loci sont pris en compte, l'expression de la différenciation devient :

$$
G_{S T}=1-H_{S} / H_{T}
$$

où $H_{S}$ et $H_{T}$ sont les moyennes (sur l'ensemble des loci) des diversités intrapopulation et totale.

\section{Paramètre de différenciation pour des caractères quantitatifs}

La variabilité phénotypique d'un caractère quantitatif peut être décomposée selon un modèle hiérarchique en une composante inter-population $\left(V_{\text {inter }}:\right.$ variance entre populations) et une composante intrapopulation $\left(V_{\text {intra }}\right.$ : variance additive moyenne sur l'ensemble des populations). La différenciation entre populations peut alors être exprimée par le rapport suivant :

$$
t=V_{\text {inter }} /\left(V_{\text {inter }}+V_{\text {intra }}\right)
$$

Pour un caractère quantitatif neutre, il est possible d'établir une relation entre entre $G_{S T}$ et $t$ (Wright, 1951, 1968; Cockerham, 1969; Rogers and Harpending, 1983). Dans le cas d'un caractère dépendant d'un locus et sans dominance, et si chaque population est en équilibre de Hardy-Weinberg :

$$
\begin{aligned}
V_{\text {inter }} & =2 G_{S T} V_{A t} \\
V_{\text {intra }} & =\left(1-G_{S T}\right) V_{A t}
\end{aligned}
$$

où $V_{A t}$ est la variance additive relative à la population théorique constituée de l'ensemble de toutes les populations, supposée en équilibre de Hardy-Weinberg.

Il en résulte :

$$
t=2 G_{S T} /\left(1+G_{S T}\right)
$$


En général, et surtout pour des faibles valeurs de différenciation, la valeur de $t$ sera toujours supérieure à celle de $G_{S T}$. Pour que les 2 paramètres $G_{S T}$ et $t$ puissent être comparés, il est suggéré de prendre $t^{\prime}$ comme coefficient de différenciation pour les caractères quantitatifs :

$$
t^{\prime}=t /(2-t)
$$

Cependant, dans la majorité des cas étudiés chez les arbres forestiers, la composante $V_{\text {intra }}$ correspond à la variance phénotypique intrapopulation et non pas à la variance additive, entraînant une sous-estimation des paramètres $t$ et $t^{\prime}$.

\section{Résultats expérimentaux sur l'organisation de la diversité}

\section{Différenciation au niveau des marqueurs moléculaires}

En moyenne, les espèces forestières manifestent des niveaux de différenciation peu élevés (tableau II) : la valeur moyenne du paramètre $G_{S T}$ est de $7 \%$ pour les gymnospermes (121 espèces) et de $11 \%$ pour les angiospermes (73 espèces) (Hamrick et al, 1992). Le niveau de différenciation est lié à la répartition de l'espèce. Les espèces endémiques sont plus différenciées (14\%) par rapport aux espèces couvrant tout un continent (3\%) (Hamrick et al, 1992). A titre d'exemple, le chêne sessile qui couvre toute l'Europe à l'exception de la Scandinavie et de la Russie, manifeste des différences de fréquences allozymiques mineures $\left(G_{S T}\right.$ de $2 \%$ sur l'ensemble de l'Europe, données non publiées). Deux hypothèses peuvent être invoquées pour interpréter cette uniformité : 1) histoire commune des populations associée à un faible taux de mutation au marqueur considéré; 2) flux géniques importants. La première hypothèse est suscitée par l'observation que de très nombreux allèles au sein d'un genre donné sont partagés par différentes espèces (Genre Quercus, Kremer et Petit, 1993; genre Pinus, Conkle, 1992). La seconde est également confortée par l'existence d'une très forte différenciation entre populations chez les espèces à aire géographique morcelée ( $P$ halepensis $G_{S T}=30 \%$, Scheller et al, 1985).

Malgré une différenciation géographique faible au niveau des allozymes, certains enzymes manifestent une variation continue en fonction d'un gradient géographique (variation clinale). Dans le cas du hêtre en Europe, une corrélation nette a été mise en évidence entre fréquences alléliques pour la peroxydase et l'altitude (Comps et al, 1987; Comps et al, 1990). Chez l'épicéa, Bergmann (1978) note une variation parallèle entre fréquences alléliques et paramètres climatiques. Chez le chêne sessile, les fréquences alléliques des aminopeptidases suivent une variation continue avec la longitude (données non publiées). Curieusement, ces différents cas de variation clinale correspondent tous à des enzymes du métabolisme secondaire, renforçant l'hypothèse de non-neutralité pour ces enzymes, même si une variation clinale peut être obtenue dans le cas de marqueurs neutres dans le cas d'équilibre dérivemigration (migration entre populations les plus proches, ou stepping stone model, Hartl et Clark, 1989). 


\section{Différenciation au niveau des caractères quantitatifs}

La littérature en génécologie des arbres forestiers est particulièrement riche en études de variabilité entre populations. Historiquement, elles constituaient la phase initiale de description des ressources génétiques. Les premiers dispositifs expérimentaux ont été installés au début de ce siècle; à l'heure actuelle, des collections de populations ont été implantées en de multiples sites (réseau multistationnel) pour toutes les espèces économiquement importantes. La variabilité a surtout été étudiée pour des caractères liés à la phénologie de la croissance, à la vigueur et à la forme et l'architecture des arbres. Malheureusement, peu d'articles présentent une estimation du coefficient de différenciation phénotypique (équations $4,5,6$ ). Dans les rares études où le paramètre $t^{\prime}$ a pu être calculé, il est souvent supérieur à $G_{S T}$ (tableau IV).

Tableau IV. Paramètres de différenciation génétique entre populations pour des caractères quantitatifs ( $t$ et $t^{\prime}$ exprimés en \%).

\begin{tabular}{|c|c|c|c|c|c|c|c|c|c|c|c|c|c|c|c|}
\hline \multirow[t]{3}{*}{ Espèce } & \multirow[t]{3}{*}{$N 1$} & \multirow[t]{3}{*}{$N 2$} & \multicolumn{4}{|c|}{ Phénologie } & \multicolumn{4}{|c|}{ Vigueur } & \multicolumn{4}{|c|}{ Forme } & \multirow[t]{3}{*}{ Réf } \\
\hline & & & \multicolumn{2}{|c|}{$D b$} & \multicolumn{2}{|c|}{ At } & \multicolumn{2}{|c|}{$H t$} & \multicolumn{2}{|c|}{$D t$} & \multicolumn{2}{|c|}{$R c$} & \multicolumn{2}{|c|}{$N b$} & \\
\hline & & & $t$ & $t^{\prime}$ & $t$ & $t^{\prime}$ & $t$ & $t^{\prime}$ & $t$ & $t^{\prime}$ & $t$ & $t^{\prime}$ & $t$ & $t^{\prime}$ & \\
\hline \multicolumn{16}{|l|}{ Gymnospermes } \\
\hline Picea abies & 15 & 4 & 8 & 4 & & & 36 & 22 & & & & & & & $\mathbf{a}$ \\
\hline Picea glauca & 57 & 8 & 1 & 5 & 5 & 3 & 2 & 1 & & & & & & & b \\
\hline Pinus nigra & 43 & 20 & & & & & 18 & 10 & & & 27 & 16 & & & c \\
\hline Pinus pinaster & 16 & 9 & & & & & 55 & 38 & 14 & 8 & 37 & 23 & 10 & 5 & d \\
\hline menziesii & 26 & 4 & 20 & 11 & & & 13 & 7 & & & & & & & e \\
\hline \multicolumn{16}{|l|}{ Angiospermes } \\
\hline $\begin{array}{l}\text { Eucalyptus } \\
\text { camaldulensis }\end{array}$ & 13 & 10 & & & & & 36 & 22 & 31 & 18 & & & & & f \\
\hline Quercus rubra & 21 & 30 & & & & & 16 & & 9 & & & & & & g \\
\hline
\end{tabular}

$N 1$ : nombre de populations étudiées; $N 2$ : âge d'évaluation du dispositif de comparaison des populations; $D b$ : date de débourrement; $A t$ : date d'arrêt de croissance; $H t$ : hauteur totale de l'arbre; $D t$ : diamètre du tronc à $1,3 \mathrm{~m} ; R c:$ rectitude du tronc; $\mathrm{Nb}:$ nombre de branches. a) Lacaze et Arbez, 1971 ; b) Li et al, 1993 ; c) Wilcox et Miller, 1976 ; d) Guyon et Kremer, données non publiées; e) Christophe et Birot, 1979; f) Siddiqui et al, 1979; g) Kriebel et al, 1989.

Dans le cas du pin maritime, nous disposons de valeurs de GST pour de nombreux marqueurs différents (protéines totales, 16 locus, $G_{S T}=17 \%$; isozymes, 8 locus, $G_{S T}=16 \%$; terpènes, 6 locus, $G_{S T}=17 \%$, Petit et al, 1993). Chez la même espèce, la valeur de $t^{\prime}$ pour la croissance en hauteur est de $38 \%$. Les valeurs de $t^{\prime}$ constituent en fait une limite inférieure, puisque la variance intrapopulation utilisée pour le calcul de $t^{\prime}$ est la variance phénotypique et non pas la variance additive. Ces chiffres illustrent l'importance de la différenciation géographique. Pour les caractères de vigueur ou de phénologie, très liés à la valeur adaptative, plus de $50 \%$ de la variabilité totale est due aux différences entre populations. Des clines 
géographiques ont pu être mis en évidence, dont certains sont presque universels pour les arbres forestiers (Wright, 1976). Les dates de débourrement et de l'arrêt de croissance varient de manière continue en fonction de la latitude ou de l'altitude d'origine de la population (Nienstaedt, 1974). La sélection naturelle est généralement invoquée pour interpréter l'importance de la différenciation géographique et la nature clinale de la variabilité. Dans certains cas le facteur du milieu responsable de la différenciation a été identifié : photopériode pour la date d'arrêt de croissance (Ekberg et al, 1979).

\section{Comparaison de la structuration géographique de la diversité et de la variabilité}

Même si, au niveau monolocus, les allozymes sont faiblement différenciés entre populations, l'utilisation de statistiques multivariables descriptives révèle généralement une structuration géographique de la diversité (El Kassaby, 1991 pour une revue) indépendamment de la prise en compte des fréquences des allèles rares. Comme des déséquilibres gamétiques entre allozymes ont rarement été mis en évidence dans les populations panmictiques forestières (Muona, 1982), il faut en déduire que la structuration géographique au niveau multilocus résulte de l'accumulation de différences minimes au niveau de chaque locus (Smouse et al, 1982). Cette structuration géographique a été comparée à l'organisation de la variabilité intraspécifique pour les caractères quantitatifs. Pour une même espèce, ces comparaisons ont été faites de 2 manières : 1) sur le même échantillon de populations; 2) sur un échantillon différent. Malheureusement les études se référant à la première approche restent en nombre limité. Deux contributions montrent cependant qu'il y a concordance entre la discrimination géographique faite aux niveaux allozymique et phénotypique. Pinus contorta est une espèce couvrant tout le Nord-Ouest américain depuis la Californie jusqu'à l'Alaska. L'analyse en parallèle de la variabilité morphologique et allozymique rend compte d'une séparation nette entre populations septentrionales et méridionales de la sous-espèce latifolia (Wheeler et Guries, 1982). Dans le cas de l'épicéa en Europe, Lagercrantz et Ryman (1990) identifient 4 grands groupes géographiques et montrent qu'il existe une corrélation entre valeurs des composantes (analyse en composantes principales) les plus explicatives pour les 2 types de caractères (allozymes et morphologie). Les études se référant à la seconde approche (Li et Adams, 1989 pour Pseudotsuga menziesii; Steinhoff et al, 1983 pour Pinus monticola) aboutissent aux mêmes conclusions.

En première analyse, la sélection naturelle pourrait être invoquée pour interpréter la concordance entre différenciation moléculaire et phénotypique. On peut remarquer tout d'abord que la concordance n'existe qu'au niveau de zones géographiques très étendues. Pour Lagercrantz et Ryman (1990), dans le cas de l'épicéa, les grands groupes correspondent aussi à des refuges putatifs de l'espèce durant le Pléistocène. L'hypothèse d' «empreinte de l'évolution», ayant conservé les complexes de gènes contrôlant isozymes et caractères phénotypiques propres aux populations refuges à la fin du tertiaire est invoquée par ces auteurs. Déjà suggérée par Wheeler et Guries (1982), elle est aujourd'hui plus généralement admise (Strauss et al, 1992). Dans le cas du chêne sessile, elle est confortée par les résultats expérimentaux relatifs au polymorphisme de l'ADN chloroplastique. Comme les chloroplastes sont préférentiellement à transmission maternelle chez les angiospermes, l'organisation 
de la diversité génétique cytoplasmique traduit les migrations des populations par graines. Deux grands groupes géographiques totalement distincts ont été reconnus sur l'ensemble de l'Europe : un groupe occidental et oriental (Petit et al, 1993). Les allozymes révèlent la même organisation, même si les différences de fréquences alléliques restent faibles à cause des flux géniques polliniques (données non publiées). Il est probable que l'Europe ait été recolonisée par 2 ensembles de populations refuges différents, l'un situé en Espagne-Italie, l'autre en Turquie ou Asie mineure.

\section{CONCLUSION}

\section{Conséquences pour la conservation des ressources génétiques}

La politique de conservation des ressources génétiques des espèces forestières s'oriente vers une conservation in situ même si des mesures de conservation ex situ sont prises pour des espèces menacées de disparition (Arbez, 1994). Cette politique repose sur le choix des populations à protéger et la définition de l'effectif à maintenir. Les conclusions des développements théoriques et les résultats expérimentaux sur la structuration génétique dans les populations d'arbres forestiers constituent 2 cadres de réponse à ces questions. La revue bibliographique qui vient d'être faite apporte des compléments d'information à cette double interrogation, mais surtout, en suscite bien d'autres.

\section{Taille des populations}

Les développements théoriques sur les tailles de populations nécessaires au maintien de la diversité et de la variabilité (Lande et Barrowclough, 1987) utilisent comme critères de polymorphisme la diversité de Nei ( $H$, équation 1 ) pour les marqueurs génétiques et la variance additive $\left(V_{A}\right.$, équation 2) pour un caractère quantitatif, ce dernier paramètre étant lui-même dépendant de la diversité moléculaire aux loci contrôlant le caractère quantitatif. Ces 2 paramètres sont-ils les plus pertinents pour l'objectif de conservation? Ils dépendent de manière très étroite des allèles les plus fréquents. Or les résultats issus des études portant sur les allozymes montrent en général que si le multiallélisme est très fréquent, les profils de fréquences alléliques sont très déséquilibrés : le nombre efficace d'allèles est généralement inférieur à 2. D'autre part, aucune donnée n'est disponible à l'heure actuelle sur les profils de fréquence alléliques aux loci contrôlant les caractères quantitatifs. Les allèles rares sont-ils nombreux et quels sont les effets associés aux allèles rares? $\mathrm{Si}$ la conservation doit prendre en compte les allèles rares, des recherches théoriques doivent être poursuivies sur les tailles nécessaires au maintien de la richesse allélique (paramètre $A$ ), à l'instar de ce qui a été fait pour $H$ et $V_{A}$.

\section{Choix des populations à conserver}

La concordance entre structuration géographique de la diversité moléculaire et l'organisation de la variabilité géographique des caractères morphologiques a suscité l'hypothèse d'«empreinte de l'évolution» dans le polymorphisme des populations actuelles. Si elle est confirmée par les analyses du polymorphisme de l'ADN cytoplasmique, elle permettra alors de circonscrire les régions géographiques colonisées 
par les mêmes populations refuges après les dernières glaciations. Cette délimitation constituerait un premier niveau d'échantillonnage des populations à conserver.

\section{RÉFÉRENCES}

Adams WT, Strauss SH, Copes DL, Griffin AR (1992) Population genetics of forest trees. Kluwer Academic Publishers, Dordrecht

Arbez M (1994) Fondement et organisation des réseaux européens de conservation des ressources génétiques forestières. Genet Sel Evol, 26, Suppl 1

Bary-Lenger A, Nebout JP (1993) Le chêne. Editions du Perron, Liège

Becker M (1984) À propos du dépérissement du chêne : réflexion sur la place actuelle de cette espèce dans la forêt française. Rev Géogr Pyrénées Sud-Ouest 55, 173-180 Bergmann F (1978) The allelic distribution at an acid phosphatase locus in Norway spruce (Picea abies) along similar climatic gradients. Theor Appl Genet 52, 57-64

Christophe C, Birot Y (1979) Genetic variation within and between populations of Douglas fir. Silvae Genet 28, 197-206

Cockerham CC (1969) Variances of gene frequencies. Evolution 23, 72-84

Comps B, Thiébaut B, Merzeau D (1991) Genetic variation in European beech stands (Fagus sylvatica L). In: Genetic variation in European populations of forest trees (Müller-Starck G, Ziehe M, eds). Sauerländer's Verlag, Frankfurt-am-Main, $110-124$

Comps B, Barrière G, Merzeau D, Letouzey J (1987) La variabilité alloenzymatique des hêtraies dans les sous-domaines medio- et eu-atlantiques d'Europe. Can J For Res 17, 1043-1049

Comps B, Thiebaut B, Paule L, Merzeau D, Letouzey J (1990) Allozymic variability in beechwoods (Fagus sylvatica L) over central Europe: spatial differentiation among and within populations. Heredity $65,407-417$

Conkle MT (1981) Proceedings of the symposium on isozymes of North American forest trees and forest insects. USDA For Serv, Gen Tech Rep PSW 48, Berkeley, California

Conkle MT (1992) Genetic diversity - seeing the forest through the trees. New Forests 6, 5-22

Copes DL (1981) Isoenzyme uniformity in western red cedar seedlings from Oregon and Washington. Can J For Res 11, 451-453

Cotterill PP, Dean CA, Van Wyk G (1987) Additive and dominance genetic effects in Pinus pinaster, $P$ radiata, $P$ elliottii and some implications for breeding strategy. Silvae Genet 36, 221-232

Duby G, Wallon A (1975) Histoire de la France rurale. Éditions du Seuil, Paris, Vol 1

Ekberg I, Eriksson G, Dormling I, (1979) Photoperiodic reactions in conifer species. Holart Ecol 2, 255-263

El Kassaby YA (1991) Genetic variation within and among conifer populations: review and evaluation of methods. In: Biochemical markers in the population genetics of forest trees (Fineschi S, Malvotti ME, Cannata F, Hattemer HH, eds). SPB Academic Publishing bv, The Hague, Hollande 
Fowler DP, Lester DT (1970) Genetics of red pine. USDA For Serv Wash Off Res Pap WO-8

Hamrick JL, Godt MJ (1990) Allozyme diversity in plant species. In : Plant population genetics, breeding, and genetic resources (Brown AHD, Clegg MT, Kahler AL, Weir BS, eds). Sinauer associates, Sunderland, 43-63

Hamrick JL, Godt MJ, Sherman-Broyles SL (1992) Factors influencing levels of genetic diversity in woody plant species. New For 6, 95-124

Harding KJ, Woolaston RD (1991) Genetic parameters for wood and growth properties in Araucaria cunninghamii. Silvae Genet 40, 232-237

Harding KJ, Kanowski PJ, Woolaston RD (1991) Preliminary genetic parameters estimates for some wood quality traits of Pinus caribaea var hondurensis in Queensland, Australia. Silvae Genet 40, 152-155

Hartl DL, Clark AG (1989) Principles of populations genetics. Sinauer associates, Sunderland, Massachusetts

Huntley B, Birks HJB (1983) An atlas of past and present pollen maps for Europe, $0-13000$ years ago. Cambridge University Press, Cambridge, UK

Kempthorne O (1957) An introduction to genetic statistics. John Wiley and sons, New York

King JN, Yeh FC, Heaman JC, Dancik BP (1988) Selection of wood density and diameter in controlled crosses of coastal Douglas fir. Silvae Genet 37, 152-157

King JN, Yeh FC, Heaman JC, Dancik BP (1992) Selection of crown form traits in controlled crosses of coastal Douglas fir. Silvae Genet 41, 362-370

Kremer A, Petit RJ (1993) Gene diversity in natural populations of oak species. Ann Sci For 50, Suppl 1, 186s-202s

Kremer A, Petit R, Zanetto A, Fougere V, Ducousso A, Wagner D, Chauvin C (1991) Nuclear and organelle gene diversity in Quercus robur and $\mathrm{Q}$ petraea. In: Genetic variation in European populations of forest trees (Müller-Starck G, Ziehe M, eds). Sauerländer's Verlag, Frankfurt am Main, 141-166

Kriebel HB, Merritt C, Stadt TH (1989) Genetics of growth rate in Quercus rubra: provenance and family effect by the early third decade in the North Central USA. Silvae Genet 37, 193-199

Lacaze JF, Arbez M (1971) Variabilité intraspécifique de l'épicéa (Picea abies Karst, partie française). Héritabilité et corrélations génétiques de quelques caractères au stade juvénile. Ann Sci For 28, 141-183

Lagercrantz U, Ryman N (1990) Genetic structure of Norway spruce (Picea abies): concordance of morphological and allozymic variation. Evolution 44, 38-53

Lande $\mathrm{R}$ (1975) The maintenance of genetic variability by mutation in a polygenic character with linked loci. Genet Res 26, 221-235

Lande R (1976) Natural selection and random genetic drift in phenotypic evolution. Evolution 30, 314-334

Lande R, Barrowclough GF (1987) Effective population size, genetic variation, and their use in population management. In: Viable populations for conservation (Soulé ME, ed). Cambridge University Press, Cambridge, 87-123

Ledig FT, Conkle MT (1983) Gene diversity and genetic structure in a narrow endemic, Torrey pine (Pinus torreyana). Evolution 37, 79-85

Ledig FT (1988) The conservation of diversity in forest trees. Bioscience 38, 471-479 
Li P, Adams WT (1989) Range-wide patterns of allozyme variation in Douglas-fir (Pseudotsuga menziesii). Can J For Res 19, 149-161

Li P, Beaulieu J, Corriveau A, Bousquet J (1993) Genetic variation in juvenile growth and phenology in a white spruce provenance-progeny test. Silvae Genet 42, $52-60$

Lynch M (1988) The rate of polygenic mutation. Genet Res 51, 137-148

Mitton JB (1992) The dynamic mating system of conifers. New For 6, 197-216

Moran GF (1992) Patterns of genetic diversity in Australian tree species. New For $6,49-66$

Mosseler A, Innes DJ, Roberts BA (1991) Lack of allozymic variation in disjunct Newfoundland populations of red pine (Pinus resinosa). Can J For Res 21, 525-528 Mousseau TA, Roff DA (1987) Natural selection and the heritability of fitness components. Heredity 59, 181-187

Müller-Starck G, Ziehe M (eds) (1991) Genetic variation in European populations of forest trees. JD Sauerländer's Verlag, Frankfurt-Am-Main

Muona O (1982) Potential causes for multilocus structure in predominantly outcrossing species. Silva Fenn 16, 107-114

Nebgen RJ, Lowe WJ (1982) Inheritance of growth, branch angle, and specific gravity in three American sycamore populations. Silvae Genet 31, 86-90

Nepveu G, Velling P (1983) Variabilité génétique individuelle de la qualité du bois chez Betula pendula Roth. Silvae Genet 32, 37-49

Nepveu G (1984) Contrôle héréditaire de la densité et de la rétractibilité du bois de 3 espèces de chêne (Quercus petraea, $Q$ robur, et $Q$ rubra). Silvae Genet 33, 110-113 Nei M (1973) Analysis of gene diversity in subdivided populations. Proc Natl Acad Sci $12,3321-3323$

Nei M (1977) F statistics and analysis of gene diversity in subdivided populations. Ann Hum Genet (London) 41, 225-233

Nei M (1987) Molecular evolutionary genetics. Columbia University Press, New York

Nienstaedt H (1974) Genetic variations in some phenological characteristics of forest trees. In: Phenology and seasonality modeling (Lieth H, ed). Springer Verlag, 389400

Petit RJ, Bahrman N, Baradat P, Kremer A (1993a) Hétérogénéité de la différenciation génétique au sein du génome nucléaire du pin maritime. Comptes rendus du séminaire du département de génétique et d'amélioration des plantes de l'INRA, Méribel 1993

Petit RJ, Kremer A, Wagner DB (1993b) Geographic structure of chloroplast DNA polymorphisms in European white oaks. Theor Appl Genet 87, 122-128

Price T, Schulter D (1991) On the low heritability of life-history traits. Evolution $45,853-861$

Rogers AR, Harpending HC (1983) Population structure and quantitative characters. Genetics 105, 985-1002

Samuel CJA (1991) The estimation of genetic parameters for growth and stem form over 15 years in a diallel cross of Sitka spruce. Silvae Genet 40, 67-72

Scheller G, Conkle MT, Griswald L (1985) Local differentiation among Mediterranean populations of Aleppo pine in their isozymes. Silvae Genet 35, 11-19 
Schoen DJ, Brown AHD (1991) Intraspecific variation in population gene diversity and effective population size correlates with the mating system in plants. Proc Natl Acad Sci 88, 4494-4497

Siddiqui KM, Khan M, Akhtar S (1979) Results of a 10 year old Eucalyptus camaldulensis Dehn. Provenance study at Peshawar. Silvae Genet 28, 24-26 Smouse PE, Spielman RS, Park MH (1982) Multiple-locus allocation of individuals to groups as a function of the genetic variation within and differences among human populations. Am Nat 119, 445-463

Steinhoff RJ, Joyce DG, Fins L (1983) Isozyme variation in Pinus monticola. Can $J$ For Res 13, 1122-1132

Strauss SH, Bousquet J, Hipkins VD, Hong YP (1992) Biochemical and molecular genetic markers in biosystematic studies of forest trees. New For 6, 125-158

Thomas BA, Spicer RA (1987) The evolution and palaeobiology of land plants. Croom Helm, Dioscorides Press, London

Turner C (1993) Geography and macroevolution. In: Evolution. A biological and plalaeontological approach (Skelton P, ed). Addison Wesley publishing company, New York, 605-667

Wheeler NC, Guries RP (1982) Population structure, genic diversity, and morphological variation in Pinus contorta Dougl. Can J For Res 12, 595-606

Whiteman PH, Dean CA, Doran JC, Cameron JN (1992) Genetic parameters and selection strategies for Eucalyptus nitens (Dean and Maiden) in Victoria. Silvae Genet 41, 77-82

Wilcox MD, Miller JT (1976) Pinus nigra provenance variation and selection in New Zealand. Silvae Genet 24, 132-140

Wright S (1951) The genetical structure of populations. Ann Eugen 15, 323-354

Wright S (1965) The interpretation of population structure by F-statistics with special regard to systems of mating. Evolution 19, 395-420

Wright S (1968) Evolution and the genetics of populations. Vol 2 The theory of gene frequencies. University of Chicago Press, Chicago

Wright J (1976) Introduction to forest genetics. Academic press, New York 